\title{
Multi-Parameter Analysis of Optimal Transitions from Chaotic to Stable Regions for Two Classes of Systems
}

\author{
Yury Talagaev ${ }^{1}$, Andrey Tarakanov ${ }^{2}$ \\ ${ }^{1}$ Balashov Institute of Saratov State University, Balashov, Russia \\ ${ }^{2}$ Borisoglebsk State Pedagogical Institute, Borisoglebsk, Russia \\ Email: shangyi@narod.ru, aft777@rambler.ru
}

Received November 16, 2012; revised December 15, 2012; accepted December 25, 2012

\begin{abstract}
The study of the parameter space of chaotic systems is complicated by its high dimensionality (multi-parametricability). Two approaches to the study of chaotic systems are presented: multi-parameter analysis and optimal suppression of chaotic dynamics. For non-autonomous chaotic systems, this is the way to compare the effectiveness of various correction parameters that provide optimal removal of irregular dynamics. For the class of autonomous chaotic systems, this is the way to investigate the optimal conditions of super-stable behavior for the chaotic system.
\end{abstract}

Keywords: Chaotic Systems; Multi-Parameter Analysis; Melnikov Method; Super-Stability

\section{Introduction}

A rather wide class of dynamic systems that demonstrate chaotic behavior is mathematically described by the systems of non-linear autonomous and non-autonomous differential equations [1-3]. As a rule, the dynamics of such systems is defined by the values of several parameters simultaneously.

A clear insight into the mechanisms of chaotic dynamics appearance (disappearance) is possible only through multi-parameter analysis of the system. It is shown in [4] that multi-parameter generalization of classical stability methods of non-linear systems is efficient. This approach is based on the studies of the peculiarities of boundaries of stability and instability areas of the system's linearized equations. Therefore it can be only partially used for researching the specific character of chaotic systems. Difficulties are caused by the multidimensionality of parametric space, the diversity of crucial situations and universal characteristics that arise on the boundary of "regular dynamics-chaos" transition [5] and high perturbation sensitivity of the systems. The means of parametrical analysis of chaotic systems are not developed enough, that's why we are often unable to fully understand how to make an optimal transition from chaotic region to the regular dynamics (stable) area in parametrical space by changing available parameters.

In this paper, we show two approaches to multi-parameter analysis of non-autonomous (dissipative nonlinear oscillators) and autonomous (Lorenz-like) chaotic systems. The paper is divided into two parts. In Part I we show, that Melnikov method $[1,6,7]$ can be applied for two-parameter analysis of chaos suppression conditions. The connections between the key parameters of the system, revealed in the process, let us make an advance in the investigation of optimal "chaos-regular dynamics" transitions. The solution for the problem of multi-parameter optimization which allows considering the possibility of achieving the state of super-stability by chaotic system is presented in Section 2.

\section{Multi-Parametrical Picture of Optimal Chaotic Dynamics Suppression in Dissipative Nonlinear Oscillators}

In this part, we present the results of a two-parametrical analysis of optimal chaotic dynamics suppression in dissipative nonlinear oscillators. As a reference model of this class of chaotic systems we chose a double-well Duffing oscillator

$$
\ddot{x}-\alpha x+\beta x^{3}=-\gamma \dot{x}+f \cos (\omega t) .
$$

Among other non-autonomous chaos models this oscillator is knows as a paradigmatic one, as it clearly demonstrates principal characteristics of a wide class of nonlinear dissipative oscillators.

\subsection{The Parametric Space Structure of a Non-Linear Dissipative Oscillator}

The parametric space of the oscillator (1) is five-dimensional $(\{\alpha, \beta\} \cup\{\gamma, f, \omega\})$ and unites two groups of parameters that play different roles in dynamics. It contains 
internal parameters $\alpha, \beta$ and parameters $\gamma, f, \omega$ that characterize the environment of the system. The multidimentionality of parametric space results in multi-parametricability of Melnikov function. Thus the function calculation for (1) gives

$$
\begin{aligned}
M(\theta) & =M(\theta, P) \\
& =A(\alpha, \beta, f, \omega) \sin (\omega \theta)-C\left(\alpha, \beta, \gamma_{0}\right),
\end{aligned}
$$

where

$$
A=\sqrt{2} \pi(\beta)^{-1 / 2} f \omega \operatorname{sech}\left(\frac{\pi \omega}{2 \sqrt{\alpha}}\right), C=\frac{4 \alpha^{3 / 2} \gamma}{3 \beta} .
$$

If $P$ is a set of possible parameter values for oscillator (1) then, using the condition of sign change for Melnikov function, we can allocate in $P: 1)$ regular dynamics region $P_{R} \subset P$, that corresponds to the values of parameters for which $A<C\left(\operatorname{sign}\left(M\left(\theta, P_{R}\right)\right)=\right.$ const $\left.) ; 2\right)$ complex dynamics region $P_{C} \subset P$, where $A>C$

$\left(\operatorname{sign}\left(M\left(\theta, P_{C}\right)\right) \neq\right.$ const $\left.) ; 3\right)$ the boundary $\tilde{P}$ (extreme values of parameters) between the regions of regular and complex dynamics ( $R C$ boundary), which is defined by the condition $A=C(M(\theta, \tilde{P})=0)$. As a starting point, in this paper we use the configuration of parameters $P_{0} \subset P_{C}$,

$$
\begin{aligned}
P_{0} & =\left\{\alpha_{0}, \beta_{0}\right\} \bigcup\left\{\gamma_{0}, f_{0}, \omega_{0}\right\} \\
& =\{1,4\} \bigcup\{0.154,0.095,1.1\}
\end{aligned}
$$

when oscillator (1) demonstrates typical chaotic behavior.

Convenient way for visual study of the structure of oscillator parametric space (1) is two-parameter analysis of the boundary of regular and complex dynamics regions.

Let's modify the equality $A=C$ first as

$$
\beta(\alpha)=\frac{8}{(3 \pi)^{2}}\left(\frac{\gamma_{0}}{f_{0} \omega_{0}}\right)^{2} \alpha^{3}\left(\operatorname{ch}\left(\frac{\pi \omega_{0}}{2 \sqrt{\alpha}}\right)\right)^{2},
$$

and then as

$$
f(\omega)=\frac{4}{3 \pi} \sqrt{\frac{\alpha_{0}^{3}}{2 \beta_{0}}} \gamma_{0} \omega^{-1} \operatorname{ch}\left(\frac{\pi \omega}{2 \sqrt{\alpha_{0}}}\right) .
$$

These dependencies give us two points of view for the study of the system. For fixed values of parameters from the group $\left\{\gamma_{0}, f_{0}, \omega_{0}\right\}$ function (2) defines the RCboundary on the plane $(\alpha, \beta)$ of internal parameters of the oscillator. Function (3) allows studying the RCboundary at fixed values $\left\{\alpha_{0}, \beta_{0}\right\}$ and $\gamma_{0}$ on the plane $(f, \omega)$ of the parameters of chaotizing perturbation.

The RC-boundary built with the use of the functions (2) and (3) is shown in Figure 1. For better understanding there were built two additional versions of the boundary (besides the initial configuration of parameters $P_{0}$ ) on the plane of parameters $(\alpha, \beta)$. These versions correspond to the changes of frequency values $\omega_{0} \pm \delta \omega, \delta \omega=0.1$ (Figure 1(a)) and to the values of perturbation amplitudes $f_{0} \pm \delta f_{0}, \delta f=0.005$ (Figure 1(b)). There are also three versions of RC-boundary on the plane of parameters $(\omega, f)$ : for the initial value $\alpha_{0}$ and two different values $\alpha_{0} \pm \delta \alpha_{0}, \delta \alpha_{0}=0.5$ (Figure 1(c)) and for $\beta_{0}$ and $\beta_{0} \pm \delta \beta_{0}, \delta \beta_{0}=0.5$ (Figure 1(d)).

The results shown in Figure 1 demonstrate that two basic groups of oscillator parameters are interdependent and reveal a special feature of RC-boundary, namely, that functions $\beta(\alpha)$ and $f(\omega)$ have their minimums. The minimums of $P_{0}$ configuration are the points

$$
\left(\alpha_{\min }, \beta_{\min }\right)=(0.329,1.447)
$$

and

$$
\left(\omega_{\min }, f_{\min }\right)=(0.764,0.055)
$$

correspondingly, while the region of complex dynamics is specified by the conditions $\beta>\beta_{\min }$ and $f>f_{\min }$. In this case variations of parameter values lead to various shifts of RC-boundary and change the position of minimum.

\subsection{Optimal Correction of Parameters Based on Melnikov Criterion}

Consider the situation when initial values of parameters $\alpha_{0}$ and $\beta_{0}$ are corrected. Having applied corrective amendments $h_{\alpha}$ and $h_{\beta}$ we will write down the oscillator (1) equation as

$$
\ddot{x}-\alpha_{0}\left(1+h_{\alpha}\right) x+\beta_{0}\left(1+h_{\beta}\right) x^{3}=-\gamma_{0} \dot{x}+f_{0} \cos \left(\omega_{0} t\right) .
$$

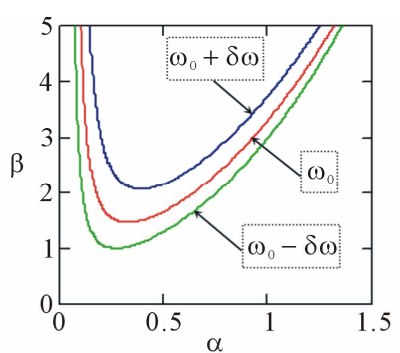

(a)

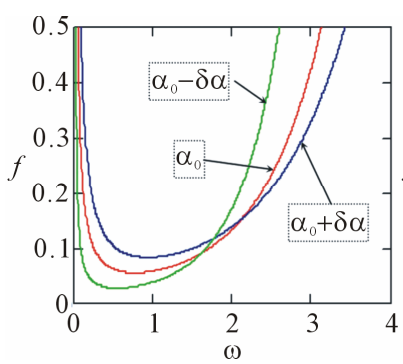

(c)

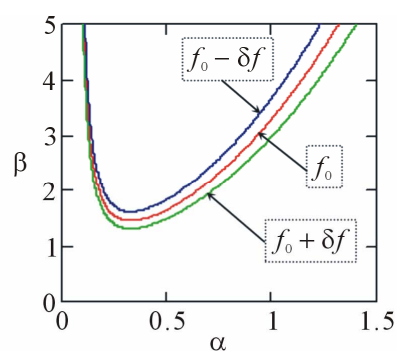

(b)

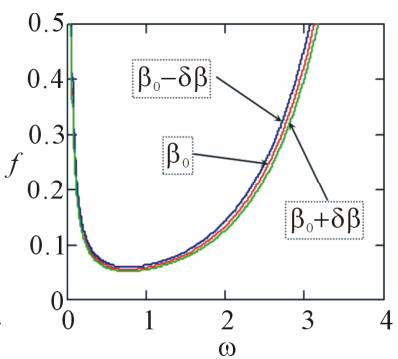

(d)
Figure 1. Two-parametric regular and complex dynamics boundary of Duffing-Holmes oscillator. 
It seems interesting to study the combined influence of amendments $h_{\alpha}$ and $h_{\beta}$ that can provide chaos suppression. As minimal corrective amendments $h_{\alpha \min }$, $h_{\beta \min }$, we can use the values that lie on the RC-boundary. With them the solution of Equation (4) will be stable instead of chaotic.

Let's define the set of accessible values $h_{\alpha}$ and $h_{\beta}$ as

$$
K=\left\{h_{\alpha}, h_{\beta} \mid M_{1}\left(\theta, P_{1}\right)=0, \forall \theta\right\}
$$

where

$$
M_{1}\left(\theta, P_{1}\right)=M_{1}\left(\theta,\left\{\alpha_{0}\left(1+h_{\alpha}\right), \beta_{0}\left(1+h_{\beta}\right)\right\} \bigcup\left\{\gamma_{0}, f_{0}, \omega_{0}\right\}\right)
$$

the Melnikov function of oscillator (4). Then, by formalizing the demand of small corrective amendments, necessary for the optimal chaos suppression, we get the optimal correction problem:

$$
h_{\alpha}^{2}+h_{\beta}^{2} \rightarrow \min _{h_{\alpha}, h_{\beta} \in K} .
$$

To solve the problem (5) we use statement (2) instead of repeating the process of calculating Melnikov function $M_{1}$. By changing $\alpha_{0} \rightarrow \alpha_{0}\left(1+h_{\alpha}\right), \beta_{0} \rightarrow \beta_{0}\left(1+h_{\beta}\right)$, we transform (2) as follows

$$
\begin{aligned}
h_{\beta}\left(h_{\alpha}\right)= & \frac{8}{(3 \pi)^{2}} \frac{1}{\beta_{0}}\left(\frac{\gamma_{0}}{f_{0} \omega_{0}}\right)^{2}\left(\alpha_{0}\left(1+h_{\alpha}\right)\right)^{3} \\
& \cdot\left(\cosh \left(\frac{\pi \omega_{0}}{2 \sqrt{\alpha_{0}\left(1+h_{\alpha}\right)}}\right)\right)^{2}-\beta_{0} .
\end{aligned}
$$

As a dependence $h_{\beta}=h_{\beta}\left(h_{\alpha}\right)$ expression (6) demonstrates the relationship between the values of corrective amendments $h_{\alpha}$ and $h_{\beta}$, that lie on the RC-boundary. Figure 2 shows the dependence $h_{\beta}=h_{\beta}\left(h_{\alpha}\right)$, the region of complex dynamics and the position of the existing optimal pair $\left(h_{\alpha \min }, h_{\beta \min }\right)$.

Actually, each point on the curve $h_{\beta}=h_{\beta}\left(h_{\alpha}\right)$ can be a solution (5), but only one of them is the best. For example, if we correct parameters $\alpha_{0}$ and $\beta_{0}$ individually, we get the pairs $\left(h_{\alpha}, h_{\beta}\right):(0.723,0)$ and $(0,-0.588)$, correspondingly. It means that if parameter $\beta_{0}$ is being corrected, the system achieves stability with smaller amendment for this criterion (5) than for $\alpha_{0}$. To find optimal pair $\left(h_{\alpha \min }, h_{\beta \text { min }}\right)$ we should find the minimum for the function $Q\left(h_{\alpha}\right)=h_{\alpha}^{2}+\left(h_{\beta}\left(h_{\alpha}\right)\right)^{2}$ while moving along the RC-boundary.

The changes of quality criterion along the RC-boundary are also shown in Figure $\mathbf{2}$ as function $Q\left(h_{\alpha}\right)$ graph. The value $h_{\alpha \min }=0.298$ corresponds to the minimum $Q\left(h_{\alpha}\right)=0.241$. Using (6) we get

$h_{\beta}\left(h_{\alpha \min }\right)=h_{\beta \min }=-0.39$. Thus the solution of optimization problem (5) gives us interrelated values of amendments $h_{\alpha \min }$ and $h_{\beta \min }$ which provide optimal transi-

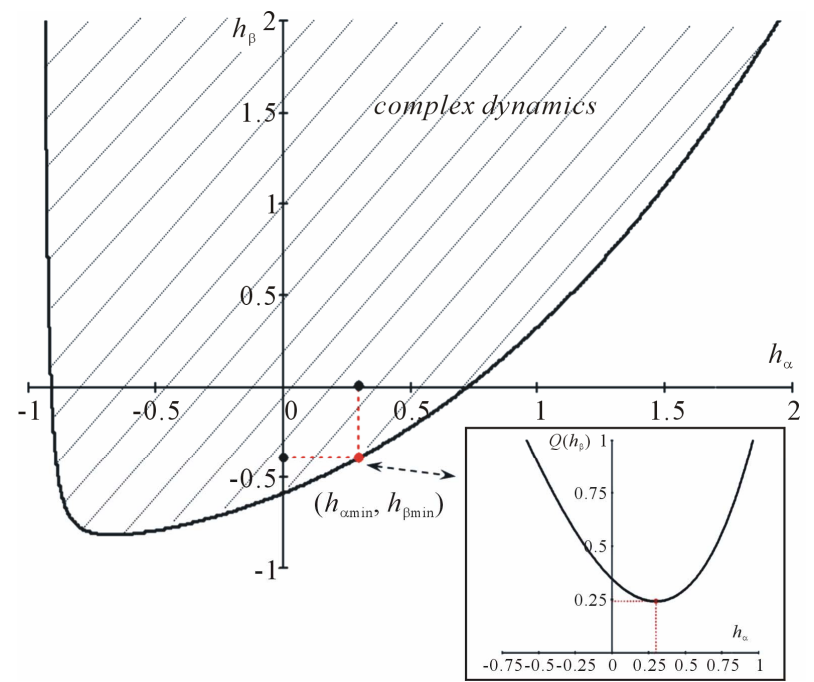

Figure 2. The boundary of the regions of regular and complex dynamics of the oscillator (6) and the position of the pair $\left(h_{\alpha \min }, h_{\beta \min }\right)$, that represents optimal combination of corrective amendments which provide chaos suppression.

tion from chaotic to stable region of parametrical space for the given configuration of parameters $P_{0} \subset P_{C}$.

\section{Super-Stability and Optimal Multiparametrical Suppression of Chaotic Dynamics in the Lorenz-Like Systems}

\subsection{Generalization of Super-Stability Conditions}

A rather wide class of chaotic Lorenz-like systems is described as follows:

$$
\dot{x}(t)=A x(t)+f(x(t)), x \in R^{3}, x(0)=x_{0}, t>0,
$$

where $A=\left(a_{i j}\right) \in R^{3 \times 3}$ - the matrix of system parameters, $f(x)$-nonlinear part $\left(f: R^{3} \rightarrow R^{3}\right)$ that characterizes nonlinear perturbation.

While suppressing chaotic dynamics, usually, we set a goal and try to find out under what conditions can the system (7) achieve stable behavior. However there exists a narrow class of systems which demonstrate a special type of regular behavior. They are super-stable systems (for essential information on super-stability see [8]). A super-stable system is always stable, but the opposite statement is not necessary true. The analysis of the superstability conditions of the system is of practical importance as the transition from unstable regime to the state of super-stability goes smoothly without an undesirable jump rise of solution norm which is possible at the initial stage of the transient process.

It is essential that, unlike stability, the super-stability is preserved at nonlinear perturbations. It grounds the possibility of analysis of super-stability achievement conditions with regard to system (7).

System (7) can have several states of equilibrium 
$x_{e}^{(k)}\left(\dot{x}_{e}^{(k)}=0\right)$, where $k=\overline{1, s}$ - the number of states of equilibrium. Then Jacobian matrix for $k$-state of equilibrium of the system (7) will be written as

$$
J_{k}=A+G_{k},
$$

where $G_{k}=\left(g_{i j}^{(k)}\right) \in R^{3 \times 3}$-is the matrix with the elements $g_{i j}^{(k)}=\left.\left(\partial f_{i}(x) / \partial x_{j}\right)\right|_{x=x_{e}^{(k)}}, i, j=1,2,3$. The generalization of super-stability condition for the systems with multiple states of equilibrium (7) leads to the condition

$$
\sigma^{(k)}=\min _{i=1, n} \sigma_{i}^{(k)}>0 \text {, }
$$

where

$$
\sigma_{i}^{(k)}=-\left(a_{i i}+g_{i i}^{(k)}\right)-\sum_{j \neq i}\left|a_{i j}+g_{i j}^{(k)}\right|, i=1,2,3 .
$$

The value $\sigma^{(k)}$ is called the super-stability degree. For every $k$-state of equilibrium the conditions $\sigma_{i}^{k}>0$ are linear restrictions on $J_{k}$ matrix coefficients that show that negative diagonal dominance should necessarily be present.

\subsection{Problem Statement}

Let initial values of parameters of the system (7) lie in the area of parametric space that corresponds to chaotic dynamics. Then according to the conditions of dynamics chaotization the Jacobian matrix $J_{k}$ of the system (7) will be unstable for every $k$ and condition (8) will not be performed.

Consider the system stabilization problem in the following form: for the given unstable matrix $J_{k}$ should be found the closest super-stable matrix $\tilde{J}_{k}$. Let's define the closeness as the least value $\left\|J_{k}-\tilde{J}_{k}\right\|$, where $\|\cdot\|$ some matrix norm (from here on $\|M\|=\left(\sum_{i, j=1}^{n} m_{i, j}^{2}\right)^{1 / 2}$ ). Then the task will be reduced to finding the optimal corrective matrix $H_{k^{*}}^{*}$ that provides super-stability of the matrix $\tilde{J}_{k^{*}}=J_{k^{*}}+H_{k^{*}}^{*}$ with condition

$$
\left\|H_{k^{*}}^{*}\right\|=\min _{k} \min _{H_{k} \in C}\left\|H_{k}\right\|,
$$

where $C$ - the set of corrective matrixes $H_{k}=\left(h_{i j}^{k}\right) \in R^{3 \times 3}$, which allow performing conditions (8) for the coefficients of the corrected matrix $\tilde{J}_{k}$.

The problem formulated above can be regarded as a generalization of the problem of static output stabilization of state space MIMO systems. The peculiarity of the problem is that we must provide not just stability, but super-stability. At the same time optimization criterion (9) is built with regard to the possibility of the system having several states of equilibrium. Hence the solution of the problem is the pair: the $H_{k^{*}}^{*}$ matrix and state of equi- librium $x_{e}^{\left(k^{*}\right)}$ correspondent to it. Together they define the corrected matrix $\tilde{J}_{k^{*}}$ which is the super-stable matrix closest to $J_{k}$.

\subsection{The Corrective Matrix Structure and Superstability Achievement Conditions}

It may seem that in problem (9) the correction rule of the matrix $J_{k}$ should be presented as transformation of its coefficients

$$
a_{i j}+g_{i j}^{(k)} \rightarrow \tilde{d}_{i j}^{k}=\left(a_{i j}+g_{i j}^{(k)}\right)+h_{i j}^{k},
$$

where $h_{i j}^{k}$-corrective amendments which define matrixes $H_{k}$ and $\tilde{J}_{k}=J_{k}+H_{k}$. However, as super-stability conditions (8) are strict there arises a question. Is there always exist the corrective matrix $H_{k}$ for the system (7), which allows fulfilling super-stability conditions?

The work [9] describes the cases when the correction of the matrix $J_{k}$ coefficients allows finding the best way to change the parameters and modify a chaotic system into the super-stable one. The structural analysis of the matrix $J_{k}=A+G_{k}$ substantionally restricts the correction rule: 1) the matrix $A$ is often sparse (that is some $a_{i j}$ are equal to zero) and its zero coefficients should not be influenced by; 2) the matrix $G_{k}$ can not be corrected at all. These restriction are caused by our attempts to apply multiparametrical correction to the system while preserving its properties. If these restrictions are not fulfilled the system undergo structural changes (new terms appear in the equation). With regard to these restrictions the correction rule will be written in the form

$$
\tilde{J}_{k}=\left(A+H_{k}\right)+G_{k}, k=\overline{1, s},
$$

where $H_{k}$ - corrective matrix structurally equivalent to $A$.

The rule (10) defines only the allowed method of correction, but does not provide us with any information about super-stability accessibility. Let's write super-stability conditions of the corrected system:

$$
\begin{gathered}
-\left(a_{i i}+h_{i i}^{(k)}\right)-g_{i i}^{(k)}>\sum_{j \neq i}\left|\left(a_{i j}+h_{i j}^{(k)}\right)+g_{i j}^{(k)}\right|, \\
i=1,2,3 .
\end{gathered}
$$

Then super-stability accessibility means that there exists matrix $H_{k}$ (according to (10)) with coefficients that satisfy these equations. It is easy to name the situations when super-stability is initially impossible. It happens when one of diagonal elements of the matrix $J_{k}$ is equal to zero. Then the existing condition (11) results in an unavoidable contradiction.

If super-stability is accessible (i.e. set $C$ in (9) is not empty) the problem (9) can be solved in two steps. At step I we should find the matrixes $H_{k}^{*}, k=\overline{1, s}$ that is to solve $s$ quadratic programming problem $\left\|H_{k}\right\|^{2} \rightarrow$ min 
with restrictions (11). At step II we find $x_{e}^{\left(k^{*}\right)}$ with matrix $H_{k^{*}}^{*}$ correspondent to it. To do this it is necessary to solve the problem $\left\|H_{k}^{*}\right\| \rightarrow \min _{k}, k=\overline{1, s}$. Once these steps are performed we get the optimal method of parametrical correction which provides super-stable dynamic behavior of the system.

Thus super-stability is a unique property peculiar to few chaotic systems. It can serve as a foundation for an additional classification (super-stabilizable systems). For example the optimal multiparametrical correction problem (9) can easily be solved for the subclass of chaotic systems investigated in [8].

$$
\dot{x}=A x+f(x)=\left(\begin{array}{ccc}
a_{11} & a_{12} & 0 \\
a_{21} & a_{22} & 0 \\
0 & 0 & a_{33}
\end{array}\right) x+x_{1}\left(\begin{array}{ccc}
0 & 0 & 0 \\
0 & 0 & -1 \\
0 & 1 & 0
\end{array}\right) x,
$$

where $x=\left(x_{1}, x_{2}, x_{3}\right)^{\mathrm{T}}$. If $a_{12} a_{21}>0, a_{12} a_{21}<0$ and $a_{12} a_{21}=0$ we get Lorenz, Chen and L ü systems, correspondingly. The existence of pairs $H_{k^{*}}^{*}, x_{e}^{\left(k^{*}\right)}$ for these systems means that the character of their dynamics can be transformed from chaotic to the stable one through parametric correction. So the systems are super-stabilizable.

\section{Conclusions}

Two results presented in the paper illustrate the effectiveness of implementation of multi-parametrical analysis for the peculiarities of chaos dynamics suppression.

First of all it is shown that Melnikov criterion can be used for stating and solving important optimization problems which deepen our understanding of chaos suppression process. While searching the optimal method of turning the system into the non-chaotic one we reveal the connection between corrective amendments to the parameters and find their minimal values.

The second result was achieved during the study of two opposite states of autonomous dynamic systemschaos and super-stability. While controlling the chaotic system it is normal to study the conditions of bringing the system to stable behavior. Still the question whether it is possible for the system to achieve super-stability remains unanswered. The conditions of achievement of the super-stable dynamics were studied for a class of Lorenz-like chaotic systems. Based on super-stability criteria the optimal multi-parametrical (matrix) correction problem was formulated and an effective method of solution was presented. The offered method allows finding super-stabilizable chaotic systems and transforming the chaotic dynamics to the super-stable one using optimal parametric correction.

Thus the named methods of multi-parametrical analysis are applied to a wide range of chaotic systems. They help compare the efficiency of different forms of parametrical perturbations and choose the ones which provide optimal suppression of chaotic dynamics.

\section{REFERENCES}

[1] S. Wiggins, "Introduction to Applied Nonlinear Dynamical Systems and Chaos," 3rd edition, Springer-Verlag, Telos, 1997.

[2] H. G. Schuster and W. Just, "Deterministic Chaos: An Introduction," 4th Edition, WILEY-VCH Verlag GmbH \& Co. KGaA, Weinheim, 2005.

[3] J. C. Sprott, "Elegant Chaos: Algebraically Simple Chaotic Flows," World Scientific, Singapore City, 2010. doi: $10.1142 / 7183$

[4] A. P. Seyranian and A. A. Mailybaev, "Multiparameter Stability Theory with Mechanical Applications," World Scientific, London, 2003. doi:10.1142/9789812564443

[5] A. P. Kuznetsov, S. P. Kuznetsov and I. R. Sataev, "A Variety of Period-Doubling Universality Classes in MultiParameter Analysis of Transition to Chaos," Physica D, Vol. 109, No. 1-2, 1997, pp. 91-112. doi:10.1016/S0167-2789(97)00162-0

[6] V. K. Melnikov, "On the Stability of the Center for Time Periodic Perturbations," Transactions of the Moscow Mathematical Society, Vol. 12, 1963, pp. 1-57.

[7] J. Guckenheimer and P. J. Holmes, "Nonlinear Oscillations, Dynamical Systems and Bifurcations of Vector Fields," Springer, New York, 1983.

[8] B. T. Polyak and P. S. Shcherbakov, "Superstable Linear Control Systems. I. Analysis," Automation and Remote Control, Vol. 63, No. 8, 2002, pp. 1239-1254. doi:10.1023/A:1019823208592

[9] Y. V. Talagaev and A. F. Tarakanov, "Superstability and Optimal Multiparametrical Suppression of Chaotic Dynamics in a Class of Autonomous Systems with Quadratic Nonlinearities," Differential Equations, Vol. 48, No. 1, 2012, pp. 153-157. doi:10.1134/S001226611110156 